\title{
A SPECTROSCOPIC SURVEY FOR $\omega$ CENTAURI MEMBERS AT AND BEYOND THE CLUSTER TIDAL RADIUS
}

\author{
G. S. Da Costa ${ }^{1}$ and Matthew G. Coleman ${ }^{1,2}$ \\ ${ }^{1}$ Research School of Astronomy \& Astrophysics, The Australian National University, Mt Stromlo Observatory, via Cotter Rd, Weston, ACT 2611, Australia; \\ gdc@mso.anu.edu.au \\ 2 Max-Planck-Institut für Astronomie, Königstuhl 17, D-69117 Heidelberg, Germany; coleman@mpia-hd.mpg.de \\ Received 2008 February 7; accepted 2008 May 5; published 2008 June 13
}

\begin{abstract}
We have used the two-degree field (2dF) multi-fiber spectrograph of the Anglo-Australian Telescope (AAT) to search for candidate members of the unusual globular cluster $\omega$ Centauri at and beyond the cluster tidal radius. Velocities with an accuracy of $\sim 10 \mathrm{~km} \mathrm{~s}^{-1}$ were obtained for 4105 stars selected to lie in the vicinity of the lower giant branch in the cluster color-magnitude diagram (CMD) and which cover an area on the sky of $\sim 2.4 \times 3.9 \mathrm{deg}^{2}$ centered on the cluster. Within the velocity interval $190-270 \mathrm{~km} \mathrm{~s}^{-1}$, the cluster member candidates have a steeply declining surface density distribution consistent with the adopted cluster tidal radius of $57^{\prime}$. For the stars in the sample beyond the tidal radius, an analysis of line strengths from the spectra, as well as radial velocities, identifies only six stars as possible candidates for extra-tidal association with the cluster. If all six of these stars are indeed related to the cluster, then a maximum of $0.7 \pm 0.2 \%$ of the total cluster mass is contained in the region between 1 and 2 tidal radii. Given this limit, we conclude that there is no compelling evidence for any significant extra-tidal population in $\omega$ Cen. The effects of tidal shocks on the outer parts of the cluster are consistent with this limit. Theories for the origin of $\omega$ Cen frequently suggest that the cluster is the former nucleus of a tidally stripped dwarf galaxy. Our results require that the stripping process must have been largely complete at early epochs, consistent with the current dynamical models of the process. The stripped former dwarf galaxy stars are therefore now widely distributed around the Galaxy.
\end{abstract}

Key words: Galaxy: stellar content - globular clusters: general - globular clusters: individual ( $\omega$ Centauri, NGC 5139) - stars: kinematics

Online-only material: machine-readable and VO tables, color figures

\section{INTRODUCTION}

The stellar system $\omega$ Centauri is unusual in many respects. When considered as part of the Galaxy's globular cluster population it is among the most luminous and the most massive, with estimates for the cluster mass ranging from 2 to $5 \times 10^{6} M_{\odot}$ (e.g., van de Ven et al. 2006; Meylan et al. 1995). Unlike other globular clusters though, $\omega$ Cen exhibits the characteristics of a system that has undergone substantial chemical self-enrichment, with its member stars showing a wide spread in abundance (e.g., Freeman \& Rodgers 1975; Norris \& Da Costa 1995; Pancino et al. 2002), and complex distributions of element-to-iron abundance ratios (see Romano et al. 2007, and the references therein). The abundance picture is further complicated by the discovery that the lower main sequence of the cluster is bimodal (Bedin et al. 2004), with the bluer sequence having a higher abundance (Piotto et al. 2005). These observations can be explained if the metal-rich but bluer sequence has a substantially higher helium abundance $(Y \approx 0.40$, see Norris 2004; Piotto et al. 2005), but generating such large helium abundances within the context of chemical evolution models is difficult (e.g., Romano et al. 2007). An extensive spectroscopic survey of $\omega$ Cen postmain-sequence members that characterizes the many and diverse properties of this cluster is presented in van Loon et al. (2007).

The dynamics of $\omega$ Cen are also unusual. Unlike most globular clusters, it has a notably flattened shape and rotates comparatively rapidly (see van de Ven et al. 2006, and the references therein). Moreover, the stellar abundances and stellar kinematics are correlated, with the more metal-rich stars being not only more centrally concentrated but also kinematically cooler (e.g., Norris et al. 1997; Sollima et al. 2005; also see
Pancino et al. 2007). Possible substructures within the cluster may also exist. For example, van de Ven et al. (2006) find evidence in their kinematic analysis for a separate disk-like component between 1 and 3 arcmin from the cluster center. Finally, the orbit of $\omega$ Cen is also unusual in that it is tightly bound and retrograde with apo- and peri-galactocentric distances of 6.2 and $1.2 \mathrm{kpc}$, respectively, and a period of $120 \mathrm{Myr}$ (Dinescu et al. 1999b). The cluster never rises very far above the Galactic plane; Dinescu et al. (1999b) give $z_{\max }$ as $1.0 \pm 0.4 \mathrm{kpc}$.

These characteristics have led to increasing support for the idea (see Freeman 1993; Norris et al. 1996, 1997) that $\omega$ Cen has not evolved in isolation, but in fact represents the nuclear remnant of a now disrupted nucleated dwarf galaxy that has been accreted by the Milky Way. Models of the orbital decay and disruption of a nucleated dwarf, which is presumed to lie initially far from the Galaxy's center, have shown that this process is dynamically plausible, with most of the dwarf's mass lost as the nucleus settles into an orbit like that of the present-day $\omega$ Cen (Bekki \& Freeman 2003; Tsuchiya et al. 2003). These dynamical models, however, do not cover a full Hubble time and while the likely present-day outcome is that the surviving nucleus is an isolated bound remnant, one may seek to confirm this prediction observationally. For example, in the model of Bekki \& Freeman (2003), the mass of the stellar envelope surrounding the nucleus, which comes from the disrupting dwarf, is still fully $\sim 50 \%$ of the mass of the nucleus at the end of the calculations $2.6 \mathrm{Gyr}$ after the initiation of the interaction. At this point, the nucleus has achieved an orbit comparable to that of $\omega$ Cen. The most likely signature of any remnant from such an interaction would be in the form of a structure beyond the tidal radius of the cluster (nucleus), probably in the form of tidal tails. 
Extra-tidal structures, particularly in the form of tidal tails, are well established around a number of globular clusters (e.g., Grillmair et al. 1995; Leon et al. 2000; Grillmair \& Johnson 2006, and references therein), with the most striking example being Pal 5 (Odenkirchen et al. 2003; Grillmair \& Dionatos 2006). The origin of these features though lies with the interaction between the cluster and the tidal field of the Galaxy rather than as a remnant from any merger process. Tidal tails have been claimed to exist in $\omega$ Cen by Leon et al. (2000) who analyzed the spatial distribution of color-magnitude diagram (CMD) selected stellar samples, chosen to maximize the contrast between the field and cluster stars in their photographic data. For $\omega$ Cen they found "two large and significant tidal tails" extending up to $\sim 2^{\circ}$ to the north and south of the cluster and estimated that the features contained up to $1 \%$ of the cluster mass. They noted, however, that dust absorption, which they did not account for, might affect the star counts. Subsequent work by Law et al. (2003) (also see M. G. Coleman, \& G. S. Da Costa 2008, in preparation), however, showed that these features are much less prominent when reddening variations are properly accounted for.

In this paper we present the results of an extensive spectroscopic survey in which we use radial velocity and line-strength information to investigate the number and distribution of $\omega$ Cen stars both within and beyond the cluster tidal radius, and to place constraints on the outer structure of the cluster and on any extra-tidal population present. The following section describes the selection of the sample, the observations, and the derivation of the radial velocities. Section 3 provides the analysis for stars both in the cluster and outside the cluster tidal radius. The main results are discussed in Section 4 and the conclusions are summarized in Section 5.

\section{OBSERVATIONS AND REDUCTIONS}

\subsection{Sample Selection}

In a forthcoming paper, M. G. Coleman \& G. S. Da Costa (2008, in preparation) will present the results of an imaging survey of a region $\sim 2.4 \times 3.9 \mathrm{deg}^{2}$ in size centered on $\omega$ Cen. The $V$ - and $I$-band photometry was obtained with the $8 \mathrm{k} \times 8 \mathrm{k}$ CCD mosaic camera mounted on the ANU 1 $\mathrm{m}$ telescope at Siding Spring Observatory. The observation, reduction, and analysis techniques employed are essentially the same as those in Coleman et al. (2005a, 2005b). Figure 1 shows a CMD from the survey for stars that lie in the radial range $10^{\prime} \leqslant r \leqslant 25^{\prime}$ from the cluster center. The principal cluster sequences such as the horizontal and giant branches, and the main-sequence turnoff, are readily visible although equally, considerable field contamination is visible, a consequence of $\omega$ Cen's relatively low galactic latitude $\left(b \approx+15^{\circ}\right)$. It is fortunate, however, that the radial velocity of the cluster is relatively large $\left(+232 \mathrm{~km} \mathrm{~s}^{-1}\right)$ permitting a generally clean separation of members from non-members via the measurement of radial velocities (e.g., Stanford et al. 2006).

A substantial radial velocity survey is required, nevertheless, in order to generate a sample of likely members in the outer parts of the cluster and beyond. The two-degree field ( $2 \mathrm{dF})$ multi-fiber instrument on the Anglo-Australian Telescope (AAT) (Lewis et al. 2002) provides an efficient way to conduct such a survey. The $2 \mathrm{dF}$ is a double-buffered system allowing the fibers for the next field to be configured while observing the current field. The reconfiguration time is of order $45-60 \mathrm{~min}$. Consequently, the

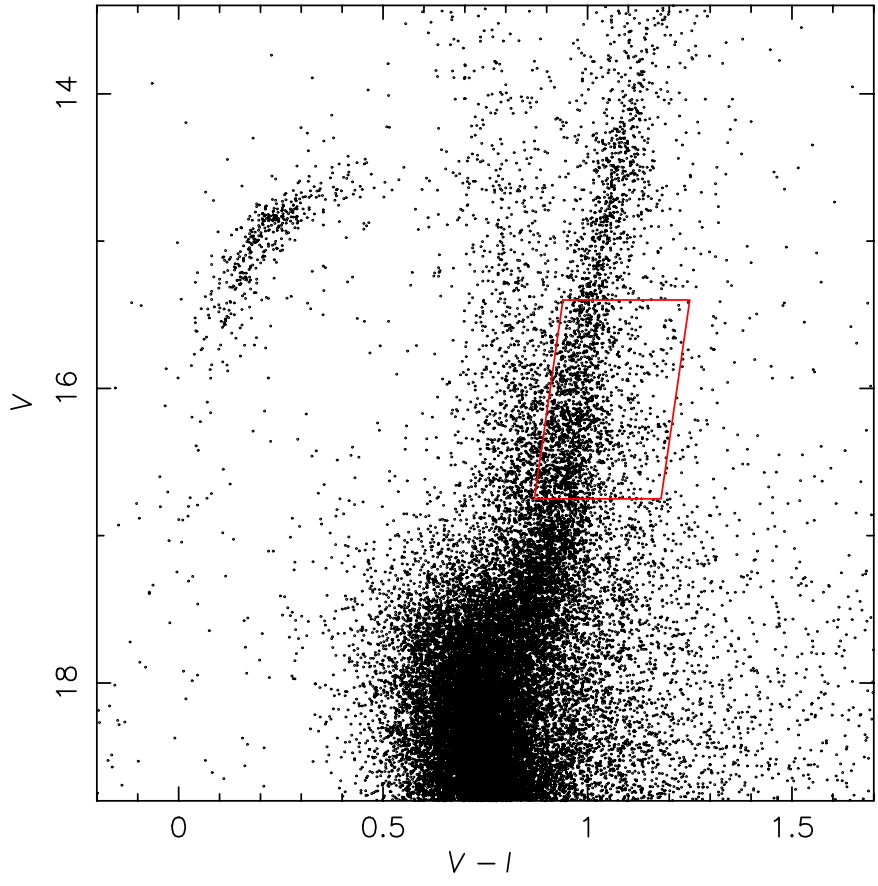

Figure 1. A CMD for stars with radial distances from the center of $\omega$ Cen in the range $10^{\prime}-25^{\prime}$ taken from the photometry of M. G. Coleman \& G. S. Da Costa (2008, in preparation). The region outlined on the cluster giant branch defines the selection box for the candidate sample observed spectroscopically.

(A color version of this figure is available in the online journal)

most efficient survey rate is achieved by selecting a magnitude interval that allows spectra with appropriate signal to noise for velocity and line-strength measurements to be obtained in total exposure times comparable to the reconfiguration time, for a given $2 \mathrm{dF}$ instrumental setup. The instrumental setup chosen used the $1200 \mathrm{~B}$ gratings centered at $\lambda 4250$ giving a wavelength coverage of approximately $\lambda \lambda 3700-4800$ at a scale of $1.1 \AA \mathrm{pix}^{-1}$. The resulting spectra have a resolution of $\sim 2.5 \AA$. These considerations led to the choice of the magnitude range $15.4 \leqslant V \leqslant 16.75$ for the initial sample selection. The corresponding color limits were then set by allowing for photometric errors and by noting that the giant branch of $\omega$ Cen has a significant intrinsic color width. The selection box is outlined in Figure 1. We note that over the area of the imaging survey, there are significant variations in the amount of interstellar reddening (see Leon et al. 2000; M. G. Coleman \& G. S. Da Costa 2008, in preparation). Consequently, the limits of the selection box were altered by the appropriate amounts given the difference in mean reddening between each CCD mosaic field and the central field. The largest shift applied was $0.08 \mathrm{mag}$ in $V-I$ and 0.18 in $V$. The initial selected sample was then modified to exclude any candidate that lay within $\sim 10^{\prime \prime}$ of a bright neighbor.

The imaging survey covers a rectangular region $\sim 2.4^{\circ}(\mathrm{EW})$ by $3.9^{\circ}$ (NS) in size while the $2 \mathrm{dF}$ field is circular. We therefore chose four $2 \mathrm{dF}$ field centers to the $\mathrm{N}, \mathrm{S}, \mathrm{E}$, and $\mathrm{W}$ of the cluster center to maximize the coverage of the imaging survey area. Stars closer to the cluster center than $20^{\prime}$ were excluded to minimize crowding concerns while still leaving enough likely members to define radial velocity and line-strength distributions. Six configurations were generated for both the $\mathrm{N}$ and $\mathrm{S}$ field centers and four for the $\mathrm{E}$ and $\mathrm{W}$ fields with a total of approximately 5500 candidates. For each field approximately 


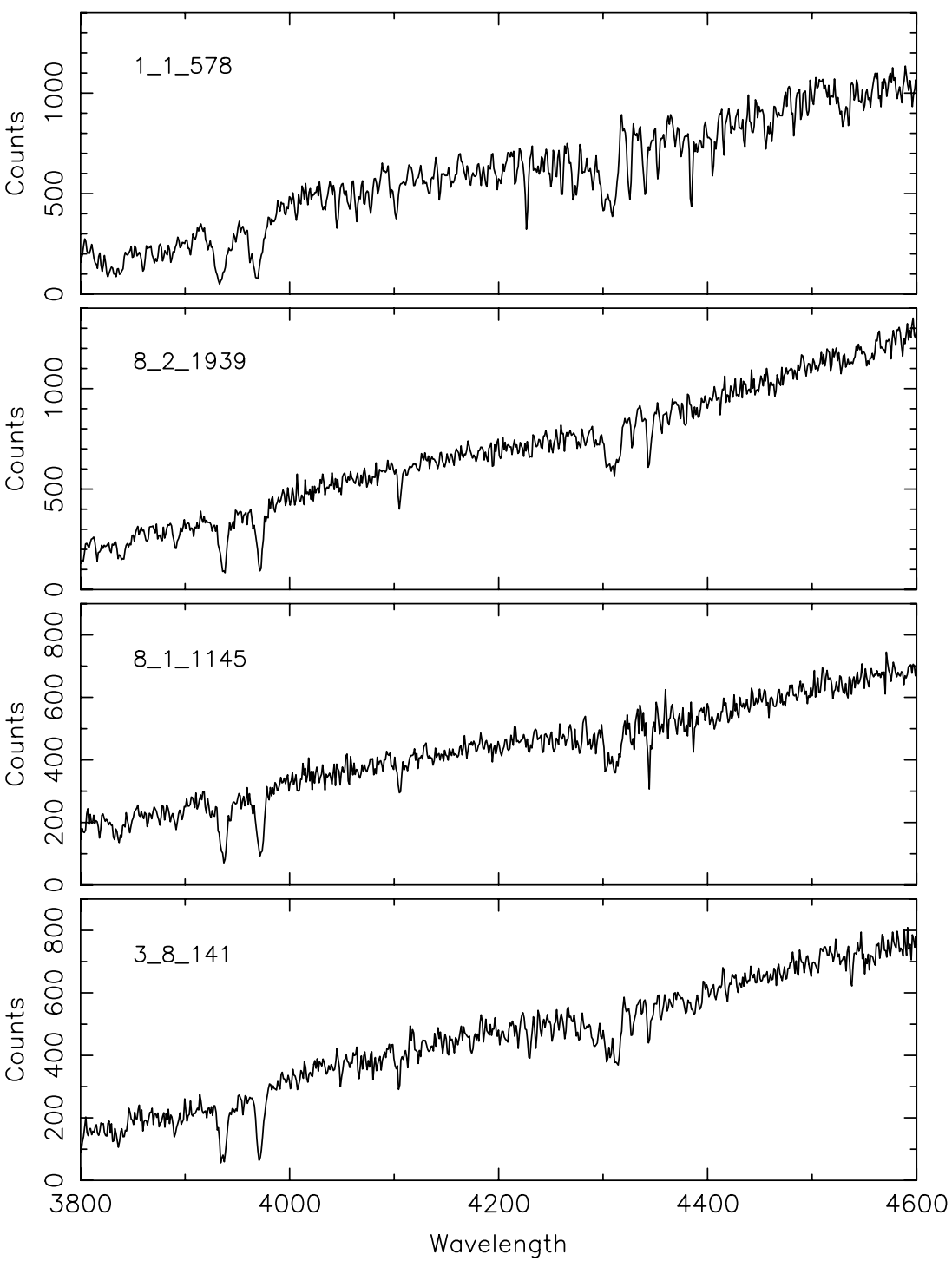

Figure 2. Examples of the reduced spectra. The uppermost spectrum is typical of the low velocity relatively metal-rich field star contaminants. The middle two spectra have radial velocities compatible with $\omega$ Cen membership and the stars lie well inside the cluster tidal radius. The lowermost spectrum is for a star with a radial velocity compatible with that for the cluster but which lies well outside the tidal radius.

15 stars were kept in common among all configurations while a modest number of stars in the overlap regions between fields were retained in the configurations for both field centers, all to allow monitoring of any systematic effects. Each configuration also included $\sim 40$ fibers allocated to blank sky regions, evenly distributed between the two $2 \mathrm{dF}$ spectrographs. These regions were selected from the imaging survey to contain no detected stars (i.e., $V \gtrsim 20$ ) within a radius of $10^{\prime \prime}$.

\subsection{Observations}

The observing run took place over 5 nights in 2003 May and a total of 16 configurations were observed: six in each of the $\mathrm{N}$ and $\mathrm{S}$ fields and two each in the $\mathrm{E}$ and $\mathrm{W}$ fields. The observing sequence for each configuration consisted of three $1800 \mathrm{~s}$ exposures preceded and followed by arc lamp exposures and a fiber flat-field exposure. Multiple exposures, including exposures recorded in each of the two $2 \mathrm{dF}$ spectrographs, were also obtained of six bright radial velocity standards. These spectra provide templates for cross-correlations with the program star spectra and for setting the velocity zero point.

The observed data sets, which come in pairs as the $4002 \mathrm{dF}$ fibers feed two separate spectrographs each imaging 200 fibers onto a CCD detector, were reduced with the $2 \mathrm{dF}$ reduction program $2 d f d r$. The fiber flat field exposure was used to generate the "tram line" map, which was then rotated and shifted to track the pixel locations of the individual fiber spectra. The fiber spectra were then "fit" extracted with the wavelength calibration coming from the appropriate arc lamp exposures. The fiber spectra for the blank sky regions were then median combined to form a single sky spectrum, which was then subtracted from each object spectrum using a weighting appropriate for the transmission of the object fiber. The relative fiber transmissions were determined either from twilight sky exposures with the same configuration or from median-combined offset sky exposures obtained as part of the observing sequence. The reduced spectra from the individual exposures for each configuration were then median combined to remove cosmic-ray contamination. Examples of the reduced spectra are shown in Figure 2. 


\subsection{Radial Velocities}

The radial velocities were determined by cross-correlation techniques using the IRAF routine $f x c o r$. The velocity analysis was carried out independently for each of the two $2 \mathrm{dF}$ spectrograph data sets, including the use of template spectra observed with the appropriate spectrograph. Only the spectra that had at least 400 counts at the $G$ band $(\sim \lambda 4300)$ were included in order to minimize the velocity errors. The primary template spectra employed were those of the $V=6.20 \mathrm{~F} 8 \mathrm{IV}-\mathrm{V}$ star HD162396, which provided the best spectral match to the program stars. The correlation was carried out over the wavelength range $\lambda \lambda 3800$ 4700 , a spectral region that encompasses a number of strong features such as the $\mathrm{Ca}$ II $\mathrm{H}$ and $\mathrm{K}$ lines, the $G$ band of $\mathrm{CH}$, and the $\mathrm{H} \delta$ and $\mathrm{H} \gamma$ hydrogen lines (see Figure 2).

Heliocentric corrections were then applied to correct for the Earth's motion resulting in velocities for 4105 program objects relative to the velocities for the two template spectra (i.e., the velocity zero points for the two data sets) which remain to be determined. These were determined through the observations of standard stars of known velocity: the zero points are set so that the mean difference between the observed and catalog velocities for the standards is zero. Six observations of four standards in addition to that of the primary template were employed for the spectrograph 1 data set, while five observations of the same four standards plus that of the primary template were used for the spectrograph 2 data. The uncertainty in the zero points is then the standard error of these mean differences. The values are $\pm 2 \mathrm{~km} \mathrm{~s}^{-1}$ for the spectrograph 1 data and $\pm 3 \mathrm{~km} \mathrm{~s}^{-1}$ for the spectrograph 2 data. The overall process was verified by making use of the eight program stars that had at least two velocity determinations in both the spectrograph 1 and the spectrograph 2 data. The mean velocity difference (spectrograph 1 - spectrograph 2) for these stars is $2 \mathrm{~km} \mathrm{~s}^{-1}$ with a standard error of the mean of $4 \mathrm{~km} \mathrm{~s}^{-1}$, indicating excellent consistency between the two data sets. The errors in the individual program star velocities were then determined from the 19 program stars that had at least five observations during the observing run, including observations across both data sets. The mean standard deviation from these sets of multiple observations, which we take as the error associated with a single program star velocity determination, is $11 \mathrm{~km} \mathrm{~s}^{-1}$; the uncertainty in this value is $\pm 1 \mathrm{~km} \mathrm{~s}^{-1}$. Figure 3 shows the spatial location of the 4105 program stars with measured velocities.

\section{ANALYSIS}

\subsection{Stars Within the Tidal Radius}

Figure 4 shows velocity histograms for all stars inside the adopted cluster tidal radius of $57^{\prime}$. This value has been taken from the compilation of Harris (1996). Given the importance of this parameter, a brief discussion is appropriate. Trager et al. (1995) list a core radius of $2.6^{\prime}$ for the cluster and a concentration index $c=1.24$, which implies a tidal radius of $45^{\prime}$. Da Costa (1979) derived similar values $\left(2.6^{\prime}, 1.22,44^{\prime}\right)$ from King (1966) model fits, but noted that the observed data indicated a larger value for the tidal radius. The best-fit dynamical models of Meylan (1987) and Meylan et al. (1995), which are a sizeable improvement over the simple King (1966) models, have tidal radii between $46^{\prime}$ and $60^{\prime}$, with the larger values showing a better fit to the outermost observed surface density data points. The data presented in Section 3.3 also imply a tidal radius consistent with the Harris (1996) value. Fortunately, none of the results of this paper depend significantly on the actual value of the cluster

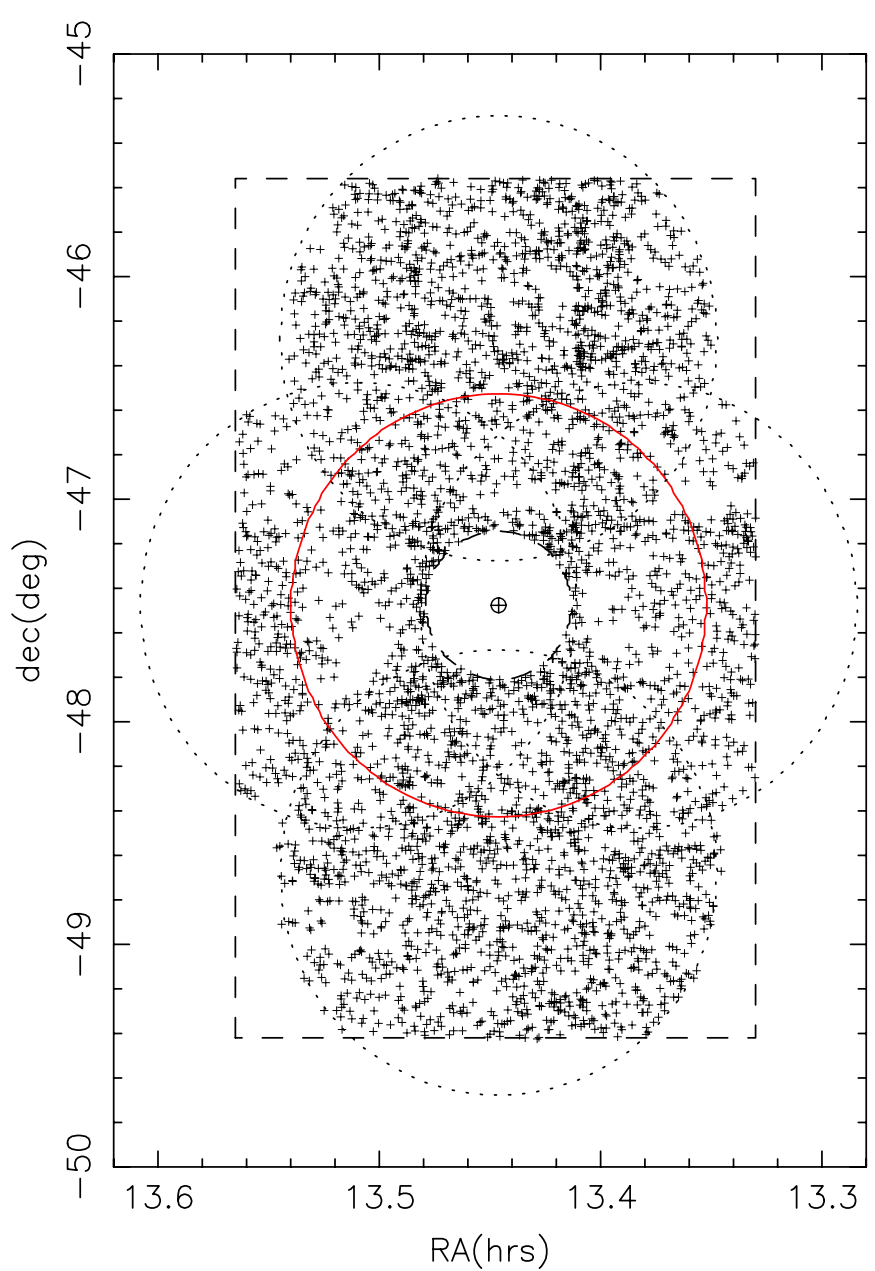

Figure 3. The location on the sky of the 4105 program stars with measured radial velocities. The rectangular region outlined with dashed lines is the extent of the imaging survey described in M. G. Coleman \& G. S. Da Costa (2008, in preparation), while the four circles marked with dotted lines outline the four $2 \mathrm{dF}$ fields. The center of the cluster is labeled with a circled plus-sign and the red circle marks the adopted cluster tidal radius of $57^{\prime}$. No stars were observed inside $20^{\prime}$ from the cluster center. Since a velocity was not determined for all possible candidates, some artifacts (mostly from the fiber allocation process) are visible in the overall distribution.

(A color version of this figure is available in the online journal)

tidal radius, provided it is similar to that adopted within a few arcmin.

The histogram in Figure 4 outlined by the solid line shows the velocity distribution for the stars with radial distances from the cluster center between $20^{\prime}$ and $30^{\prime}$. It is clearly bimodal with one population, predominantly foreground disk stars, centered near zero velocity and a second population, predominantly cluster members, centered near the cluster velocity of $232 \mathrm{~km} \mathrm{~s}^{-1}$ (e.g., van de Ven et al. 2006). The mean velocity for the 119 stars within the velocity interval 190-270 $\mathrm{km} \mathrm{s}^{-1}$ (chosen to encompass all likely members given the distribution in Figure 4) is $229 \mathrm{~km} \mathrm{~s}^{-1}$ with a dispersion of $14 \mathrm{~km} \mathrm{~s}^{-1}$. The mean velocity agrees well with the known cluster velocity given the uncertainty in the velocity system zero point discussed above. Moreover, the observed dispersion value is consistent with that expected from the combination of the intrinsic dispersion of the cluster at these radii, $\sim 8 \mathrm{~km} \mathrm{~s}^{-1}$ (see van de Ven et al. 2006), and the individual velocity errors. These stars will be referred to as the inner cluster sample.

The histogram outlined by the dot-dash line in Figure 4 is for stars with radial distances between $30^{\prime}$ and $57^{\prime}$. Because 


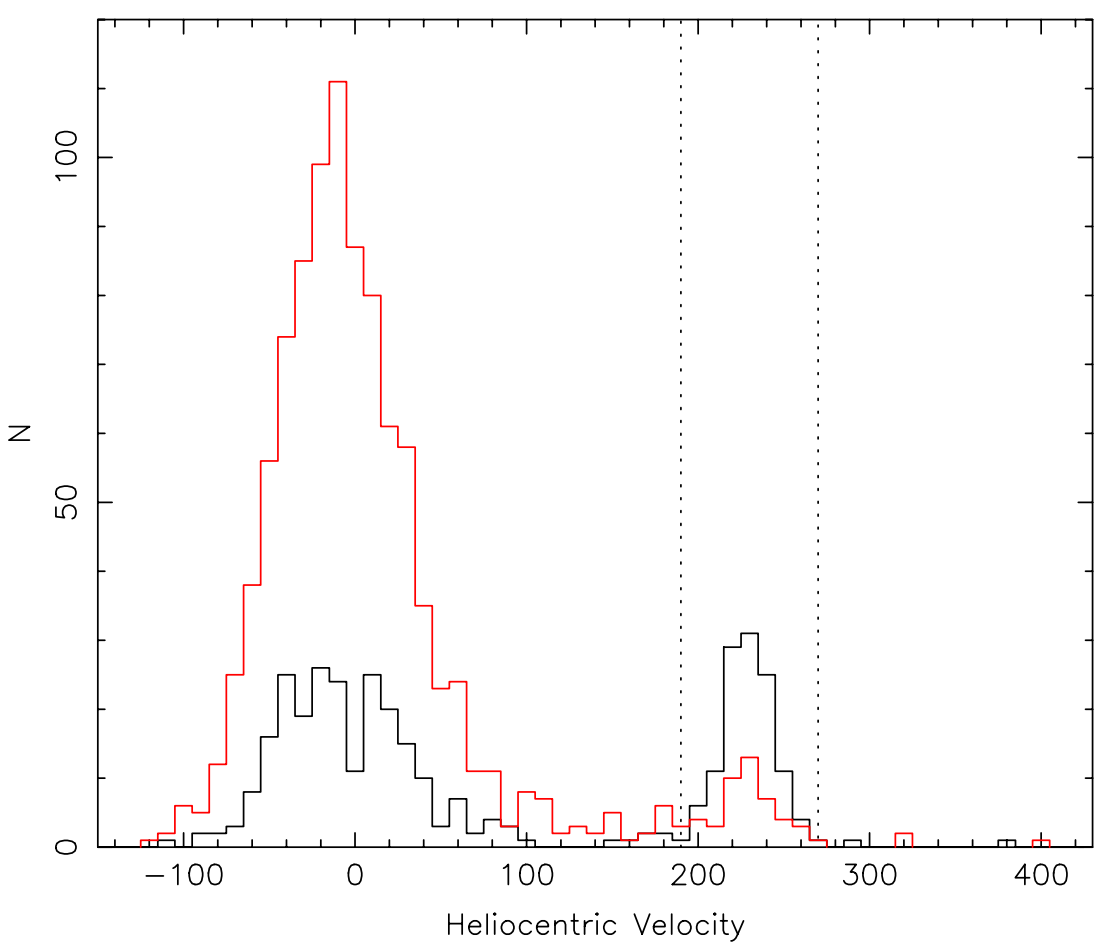

Figure 4. Velocity histograms for stars within $57^{\prime}$ of the center of $\omega$ Cen. The black line outlines the velocity distribution for stars with $20^{\prime} \leqslant r^{\prime} \leqslant 30^{\prime}$ and the red line outlines the distribution for stars with $30^{\prime} \leqslant r^{\prime} \leqslant 57^{\prime}$. The systemic velocity of the cluster is $232 \mathrm{~km} \mathrm{~s}^{-1}$ and the dotted vertical lines indicate the velocity interval from which candidate cluster members are selected.

(A color version of this figure is available in the online journal)

of the larger area and the decreasing density of cluster stars with increasing radial distance, the contaminating population now considerably outweighs the cluster population, which nevertheless remains detectable above the high velocity tail of the field star distribution. Using the same selection interval as for the inner region, the 46 candidate cluster members have a mean velocity of $227 \mathrm{~km} \mathrm{~s}^{-1}$ and a velocity dispersion of $17 \mathrm{~km} \mathrm{~s}^{-1}$. The increased velocity dispersion over that seen for the inner region presumably suggests that this outer sample is contaminated to some extent with non-cluster members. ${ }^{3}$ These stars will be referred to as the outer cluster sample.

Two other criteria can be used to investigate the question of cluster membership. First, noting that the expected proper motion of cluster members is small (cf. Dinescu et al. 1999a), we can use published proper motion catalogs to weed out from the sample any stars with significant motions, as they are unlikely to be cluster members (though equally a small proper motion does not guarantee cluster membership). Second, while it is well established that $\omega$ Cen has a substantial internal abundance range, we can still use the spectra to define relations between various line-strength parameters and again seek outliers from the relations defined by the majority of stars.

Considering first proper motions, since the majority of the candidates are too faint to be included in the UCAC2 catalog (Zacharias et al. 2004), we extracted from the SuperCOSMOS Science Archive 4 proper motions for all the stars in the outer cluster sample (due to crowding effects, we did not consider the proper motions for the inner cluster sample). Hambly et al. (2001) indicate that for the magnitude range of our program stars, the estimated proper motion accuracy is of order

\footnotetext{
3 We have been allocated additional time on the AAT to measure more precise velocities for stars in this sample, in order to improve the constraints on the velocity dispersion in the outer parts of the cluster.

4 http://surveys.roe.ac.uk/ssa/index.html.
}

Table 1

Spectral Feature and Continuum Bandpasses

\begin{tabular}{lccc}
\hline \hline Feature & $\begin{array}{c}\text { Feature bandpass } \\
(\AA)\end{array}$ & $\begin{array}{c}\text { Blue continuum } \\
\text { bandpass }(\AA)\end{array}$ & $\begin{array}{c}\text { Red continuum } \\
\text { bandpass }(\AA)\end{array}$ \\
\hline Ca II K & $3925-3943$ & $3909-3921$ & $4010-4030$ \\
H $\delta$ & $4093-4109$ & $4010-4030$ & $4120-4131$ \\
$G$-band $(\mathrm{CH})$ & $4294-4316$ & $4230-4248$ & $4415-4435$ \\
$\mathrm{H} \gamma$ & $4334-4347$ & $4230-4248$ & $4415-4435$ \\
\hline
\end{tabular}

10 mas $\mathrm{yr}^{-1}$. The mean motions in right ascension (R.A.) and declination (decl.) for the 46 stars were calculated along with the standard deviations, which were $6.3 \mathrm{mas} \mathrm{yr}^{-1}$ in R.A.

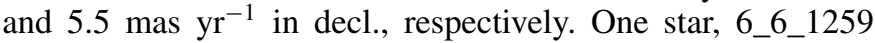
(Table 3), lay outside the error ellipse defined by $3 \sigma_{\mu, \alpha}$ and $3 \sigma_{\mu, \delta}$. It was not considered in the analysis of spectral line strengths.

For line-strength parameters, we first removed the overall continuum shape of the spectra by fitting a lower order polynomial using the IRAF routine continuum. Following, for example, Beers et al. (1999), we then measured via numerical integration the equivalent widths of the $\mathrm{Ca}$ II $\mathrm{K}$ line and the $G$ band of $\mathrm{CH}$ and via Gaussian fits the equivalent widths of the $\mathrm{H} \delta$ and $\mathrm{H} \gamma$ hydrogen lines (Figure 2). The continuum and feature bandpasses used are listed in Table 1. The K-line and $G$-band strengths serve as metal abundance indicators, while the hydrogen line strengths serve as an indicator of effective temperature. The mean uncertainties in the line strengths were then determined from the standard deviations of the measurements for the seven stars in the inner sample that have at least six observations. For stars with multiple spectra, the individual line-strength measurements were averaged. For one star, it was not possible to measure all four indices: for star 8_6_15256, the $\mathrm{H} \delta$ line was significantly affected by noise and no reliable 

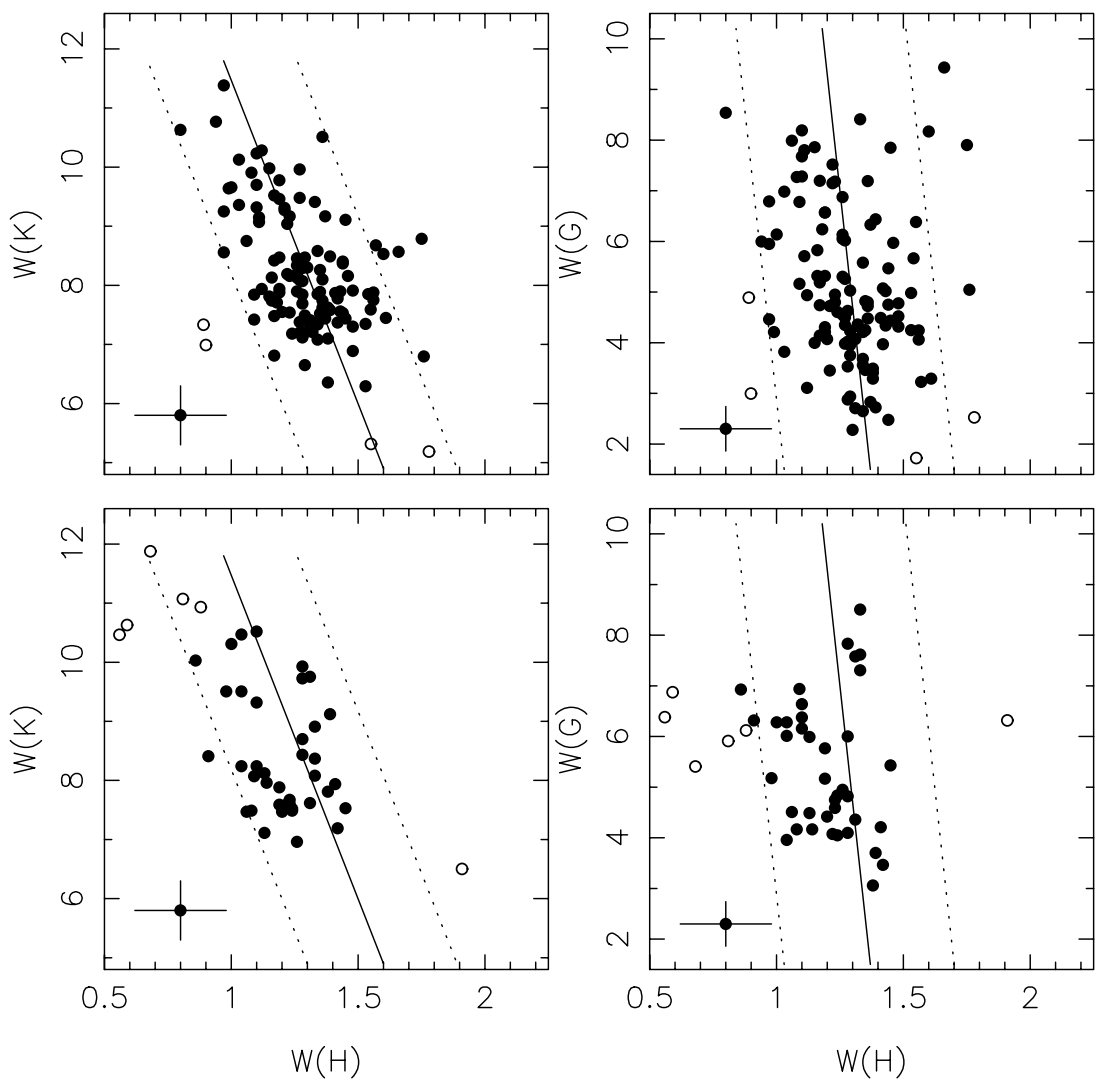

Figure 5. Line-strength measurements. The left panels show the equivalent width of the $\mathrm{Ca}$ II $\mathrm{K}$ line plotted against the mean equivalent width of the $\mathrm{H} \delta$ and $\mathrm{H} \gamma$ hydrogen lines. The right panels plot the $G$-band $(\mathrm{CH})$ strength against the hydrogen line strength. The upper panels are for the inner sample while the lower panels are for the outer sample. Open circles represent stars that are classified as probable non-members of the cluster. The solid lines are least-squares fits to the inner sample, with the dotted lines representing $\pm 2 \times$ the rms about the fit. The fits are reproduced in the lower panels. Mean error bars are shown in the bottom-left corner of each panel.

measurement could be made. The other line strengths, however, are consistent with cluster membership.

Given that the inner sample is likely to consist largely of cluster members, we used this sample to define relations between the $\mathrm{K}$-line strength and the average hydrogen line strength $W_{H}=\left[W(H)_{\delta}+W\left(H_{\gamma}\right)\right] / 2$, and between the $G$-band strength and $W_{H}$ for probable cluster stars. These relations are shown in the upper panels of Figure 5. Turning first to the upper left panel, we can see that, as expected given the wellestablished abundance range in $\omega$ Cen, there is a significant range in the $\mathrm{K}$-line strengths for the inner sample stars that is anti-correlated with hydrogen line strength. The solid line in the panel represents a least-squares fit to the data, while the dotted lines represent $\pm 2 \times$ the rms about the fit after excluding the four stars plotted as open circles. Two of these are clearly deviant, but exclusion of the two stars with the weakest $\mathrm{K}$ lines deserves some comment. The abundance distribution in $\omega$ Cen is well established (e.g., Norris et al. 1996) and is characterized by a steep initial rise to the mean abundance followed by a long tail to higher abundances. Excluding the two stars with the lowest K-line strengths, the distribution of K-line strengths in the panel reflects this general shape and for that reason the two stars have been excluded. A small number of stars also lie to the right of the $+2 \sigma$ line in the panel. These are nevertheless considered members because they all show very strong $G$-bands and other features indicating probable carbon overabundances (see Figure 6). The exclusion of four stars from the inner sample as possible non-members is consistent with the number of field stars expected in the adopted cluster velocity range, given the number of stars in the velocity intervals adjacent to that for the cluster seen in the solid histogram in Figure 4.

The upper right panel of Figure 5 shows the distribution of $G$-band strengths for the inner sample. Once again the solid line is a least-squares fit and the dotted lines are $\pm 2 \times$ the rms about the fit after again excluding the four stars plotted as open circles. There is clearly a very large range in $G$-band strengths for the stars in this sample. Many of the stars with strong $G$ bands also show additional $\mathrm{CH}$ absorption in the region around $\lambda 4370$; some examples are shown in Figure 6. The existence of stars in $\omega$ Cen with a range of overabundances of carbon is well established; see, for example, Stanford et al. (2007) and references therein.

The lower left panel shows $W_{K}, W_{H}$ for the stars in the outer sample. The solid and dotted lines are reproduced from the equivalent panel for the inner sample. Given that there is evidence for a radial abundance gradient in the cluster, in the sense that metal-rich objects are less frequent in the outer regions (e.g., Norris et al. 1996), it seems unlikely that the outer sample would contain comparatively more metal-rich objects than the inner sample. Consequently, for the stars with strong K-lines, we have considered stars with $W_{K} \gtrsim 10.6 \AA$ to be non-members even if they are within the $\pm 2 \sigma$ boundaries. In many cases these stars have $G$-band strengths that fall outside the $\pm 2 \sigma$ boundary in the lower right panel, confirming their probable non-member status. All in all, we classify six of the outer sample stars as non-members. As noted above, there is a seventh probable non-member in the outer sample based on a discrepant proper motion. Interestingly, like the inner region, the outer region also 


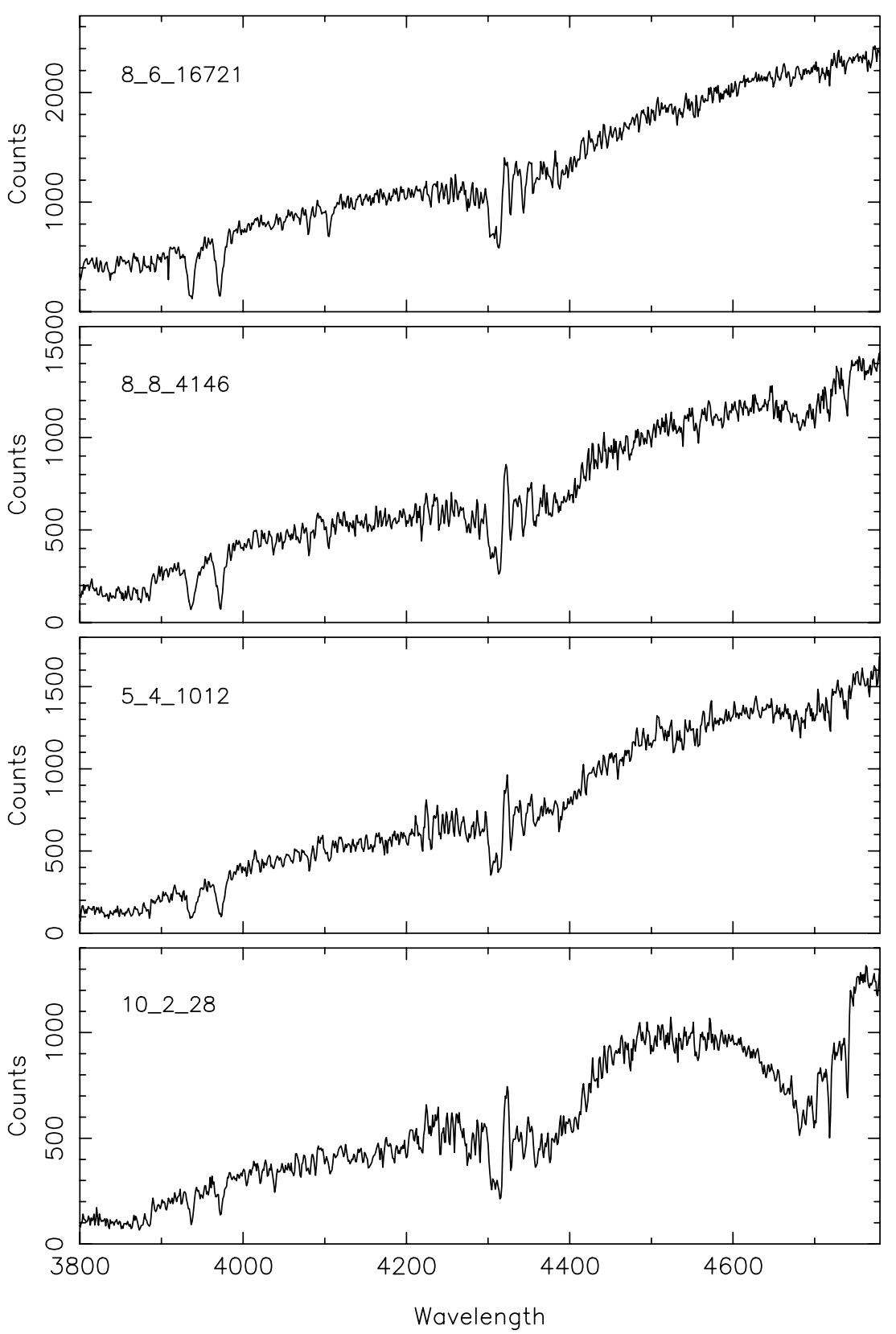

Figure 6. Spectra of stars showing enhanced carbon features at the $G$-band, $\lambda \approx 4300 \AA$; at additional $\mathrm{A}-\mathrm{X} \mathrm{CH}$ bands, $\lambda \approx 4370 \AA$; and at $\mathrm{C}_{2}$ bands, $\lambda \approx 4700 \AA$. The upper two stars come from the inner sample while the third is from the outer sample. The star in the bottom panel is clearly a carbon star. Its membership status is discussed in the text.

contains a number of $\mathrm{CH}$-strong stars. Examples are shown in Figure 6. Inspection of the dashed line histogram in Figure 4 suggests that excluding seven stars from the outer sample as likely non-members is perhaps a conservative number; there may well be an additional number of approximately the same size of non-member stars in this sample that we have not been able to conclusively identify. Such identification would require velocities with a higher degree of precision than that presented here as well as knowledge of the cluster velocity dispersion in this outer region.

One star deserves special mention. This is star 10_2_28 whose spectrum is shown in the bottom panel of Figure 6. With strong $\mathrm{C}_{2}$ features at $\lambda \approx 4700 \AA$, strong $\mathrm{CH}$ absorption features in the region $\lambda \lambda 4250-4430$, and strong $\mathrm{CN}$ absorption features at $\lambda \approx$ $3883 \AA$ and $\lambda \approx 4215 \AA$, this star is clearly a carbon star. Such stars are not unknown in $\omega$ Cen (e.g., van Loon et al. 2007) but the known member stars with similar spectra, such as ROA 577 (see Figure 25 of van Loon et al. 2007), are found on the red giant branch at considerably brighter magnitudes. Determining the radial velocity of 10_2_28 is not straightforward given the very different spectral type. Nevertheless, cross-correlations with the latest spectral type among the observed radial velocity standards and with probable $\omega$ Cen members such as 8_8_4146 (Figure 6) indicate a velocity of $\sim 260 \pm 15 \mathrm{~km} \mathrm{~s}^{-1}$. This is nominally within the velocity range of probable cluster members, but the star lies at a distance of $47.3^{\prime}$ from the cluster center. Given that the number of cluster members at this radius is small, and that cluster members of this type are comparatively rare, we conclude that this star is likely not a member of the cluster. Interestingly, there is a second carbon star that lies in the field of the cluster but which is clearly not a cluster member (ROA 153; see Smith \& Wing 1973). 
Table 2

Probable Cluster Member Stars

\begin{tabular}{lccccccc}
\hline \hline ID & $\begin{array}{c}R \\
(\operatorname{arcmin})\end{array}$ & $\begin{array}{c}\text { Velocity } \\
\left(\mathrm{km} \mathrm{s}^{-1}\right)\end{array}$ & $N$ & $\begin{array}{c}\text { R.A. } \\
(\mathrm{J} 2000)\end{array}$ & $\begin{array}{c}\text { Decl. } \\
(\mathrm{J} 2000)\end{array}$ & $V$ & $V-I$ \\
\hline 8_4_3206 & 20.0 & 239 & 1 & 132747.3 & -474544 & 16.42 & 0.97 \\
8_7_15831 & 20.1 & 224 & 1 & 132506.5 & -471742 & 15.80 & 0.96 \\
8_6_16385 & 20.1 & 219 & 1 & 132456.3 & -473635 & 16.39 & 0.93 \\
8_8_3134 & 20.2 & 221 & 2 & 132547.7 & -471100 & 16.63 & 0.89 \\
8_6_15256 & 20.2 & 197 & 1 & 132511.4 & -474103 & 16.66 & 0.90 \\
8_8_2219 & 20.2 & 228 & 1 & 132604.4 & -470940 & 16.42 & 1.02 \\
8_4_4900 & 20.3 & 248 & 1 & 132720.0 & -474803 & 16.23 & 0.97 \\
8_2_1336 & 20.3 & 253 & 1 & 132843.3 & -472438 & 16.19 & 1.00 \\
7_3_261 & 20.3 & 222 & 1 & 132446.0 & -473014 & 16.68 & 1.07 \\
5_4_1514 & 20.4 & 209 & 1 & 132709.5 & -470834 & 16.47 & 0.93
\end{tabular}

(This table is available in its entirety in machine-readable and Virtual Observatory (VO) forms in the online journal. A portion is shown here for guidance regarding its form and content.)

Table 3

Probable Non-Cluster Member Stars

\begin{tabular}{lccccccc}
\hline \hline ID & $\begin{array}{c}R \\
(\operatorname{arcmin})\end{array}$ & $\begin{array}{c}\text { Velocity } \\
\left(\mathrm{km} \mathrm{s}^{-1}\right)\end{array}$ & $N$ & $\begin{array}{c}\text { R.A. } \\
(\mathrm{J} 2000)\end{array}$ & $\begin{array}{c}\text { Decl. } \\
(\mathrm{J} 2000)\end{array}$ & $V$ & $V-I$ \\
\hline 7_2_312 & 20.7 & 197 & 2 & 132444.4 & -472556 & 16.21 & 0.96 \\
11_1_4329 & 21.4 & 226 & 1 & 132727.7 & -474847 & 16.53 & 0.96 \\
8_1_1381 & 22.5 & 232 & 1 & 132824.3 & -471333 & 16.55 & 0.88 \\
11_8_1684 & 23.0 & 232 & 1 & 132600.2 & -475014 & 16.06 & 1.04 \\
11_1_3775 & 32.5 & 219 & 1 & 132739.0 & -475955 & 15.56 & 1.22 \\
5_6_92 & 34.9 & 202 & 1 & 132636.1 & -465348 & 16.06 & 1.03 \\
12_8_1988 & 37.9 & 206 & 1 & 132930.5 & -475425 & 16.35 & 1.15 \\
6_6_1259 & 45.0 & 197 & 2 & 132956.3 & -465716 & 16.73 & 0.89 \\
10_2_28 & 47.3 & 260 & 1 & 132445.8 & -481124 & 16.17 & 1.14 \\
10_1_2777 & 48.0 & 254 & 1 & 132231.9 & -475017 & 16.43 & 0.88 \\
4_3_2022 & 54.6 & 256 & 2 & 132307.1 & -464842 & 16.15 & 1.21 \\
11_3_191 & 55.1 & 243 & 1 & 132917.7 & -481731 & 15.54 & 1.16 \\
\hline
\end{tabular}

Notes. Star 6_6_1259 excluded on the basis of discrepant proper motion; all others excluded on the basis of discrepant line strengths; star 10_2_28 is a carbon star.

In Table 2 we list the ID number, the radial distance from the cluster center, the right ascension and declination, the number of observations, the radial velocity, and the $V$ and $V-I$ photometry from M. G. Coleman \& G. S. Da Costa (2008, in preparation) for the 154 probable $\omega$ Cen members categorized in this work. At a distance of $54.7^{\prime}$ from the cluster center, star 9_4_1918 is the most radially distant probable cluster member inside the adopted tidal radius; there are nine such stars beyond $40^{\prime}$ from the cluster center. For completeness in Table 3 we list the same information for the 12 stars in the inner and outer cluster samples that are classified as probable non-members, including the carbon star 10_2_28, despite having radial velocities comparable to that for the cluster. Similarly, Table 4 lists the same information for the 1183 stars inside the adopted cluster tidal radius that have velocities outside the velocity interval $\left(190-270 \mathrm{~km} \mathrm{~s}^{-1}\right)$ used to select cluster member candidates.

Figure 7 shows the photometry for the probable members superposed on the photometry for all stars in the $10^{\prime}-25^{\prime}$ radius region (Figure 1). As expected given the cluster metallicity distribution, the blue side of the color distribution of the probable members is sharply defined but there is a considerable spread to redder colors.

\subsection{Stars Beyond the Tidal Radius}

In Figure 8, we show the velocity distribution for stars beyond the adopted tidal radius. The distribution is clearly dominated
Table 4

Non-Member Stars

\begin{tabular}{lcrccccc}
\hline \hline ID & $\begin{array}{c}R \\
(\operatorname{arcmin})\end{array}$ & $\begin{array}{c}\text { Velocity } \\
\left(\mathrm{km} \mathrm{s}^{-1}\right)\end{array}$ & $N$ & $\begin{array}{c}\text { R.A. } \\
(\mathrm{J} 2000)\end{array}$ & $\begin{array}{c}\text { Decl. } \\
(\mathrm{J} 2000)\end{array}$ & $V$ & $V-I$ \\
\hline 11_8_135 & 20.0 & -11 & 2 & 132644.3 & -474838 & 16.05 & 1.02 \\
8_4_4179 & 20.2 & -42 & 1 & 132731.3 & -474716 & 16.43 & 1.02 \\
11_1_5863 & 20.2 & 34 & 10 & 132659.0 & -474844 & 16.23 & 1.08 \\
8_8_1220 & 20.5 & -5 & 8 & 132621.6 & -470830 & 16.39 & 0.85 \\
8_1_1736 & 20.6 & -11 & 1 & 132814.9 & -471439 & 16.24 & 0.94 \\
8_2_2607 & 20.6 & 168 & 1 & 132823.8 & -471618 & 15.67 & 1.05 \\
8_8_2721 & 20.6 & 26 & 1 & 132555.6 & -470952 & 16.65 & 0.95 \\
8_5_4726 & 20.7 & 6 & 1 & 132517.3 & -474302 & 16.29 & 1.03 \\
8_8_4580 & 20.7 & -19 & 1 & 132512.7 & -471509 & 16.64 & 0.97 \\
5_4_1370 & 20.9 & 55 & 8 & 132719.0 & -470830 & 16.19 & 0.99
\end{tabular}

(This table is available in its entirety in machine-readable and Virtual Observatory (VO) forms in the online journal. A portion is shown here for guidance regarding its form and content.)

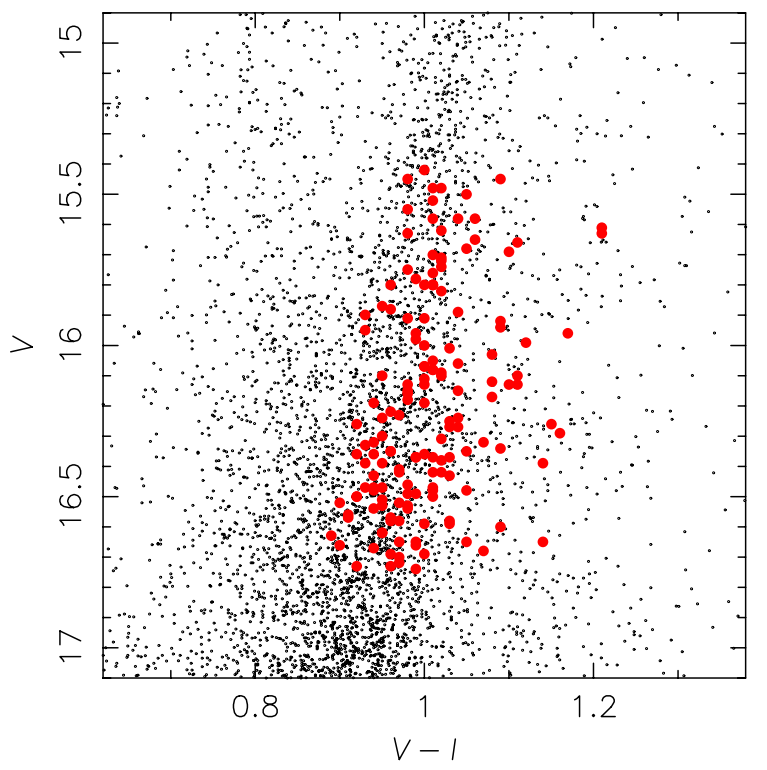

Figure 7. The $V, I$ photometry for the 154 probable members of $\omega$ Cen superposed on the photometry from Figure 1.

(A color version of this figure is available in the online journal)

by disk stars which have heliocentric velocities near zero (for reference, the zero velocity in the galactic rest frame at the Sun's location corresponds to a heliocentric velocity of $168 \mathrm{~km} \mathrm{~s}^{-1}$ in the direction of $\omega$ Cen, assuming a galactic rotation velocity of $220 \mathrm{~km} \mathrm{~s}^{-1}$ ). The inset in the figure shows a close-up of the distribution in the vicinity of the velocity of the cluster. While the numbers of stars are small and therefore subject to significant statistical fluctuation, there is no obvious indication of an excess of stars at or near the cluster velocity.

Nevertheless, there are 28 stars in the $\pm 40 \mathrm{~km} \mathrm{~s}^{-1}$ velocity range shown in the inset in Figure 8. Of these, two have proper motions inconsistent with association with $\omega$ Cen and all (including the stars with discrepant proper motions) but nine occupy locations in the $\left(W_{K}, W_{H}\right)$ and $\left(W_{G}, W_{H}\right)$ diagrams that are inconsistent with $\omega$ Cen membership. Most of these likely non-member stars have relatively large values of $W_{K} ; 18$ have $W_{K} \geqslant 9.5 \AA$ A. They are most likely members of the Galaxy's bulge population lying at distances beyond that of $\omega$ Cen. The line of sight through the cluster has a minimum distance of $10.4 \mathrm{kpc}$ from the Galactic center at a height of $3.4 \mathrm{kpc}$ above the Galactic plane. At this distance the stars would have $M_{V} \approx+0.5$, 


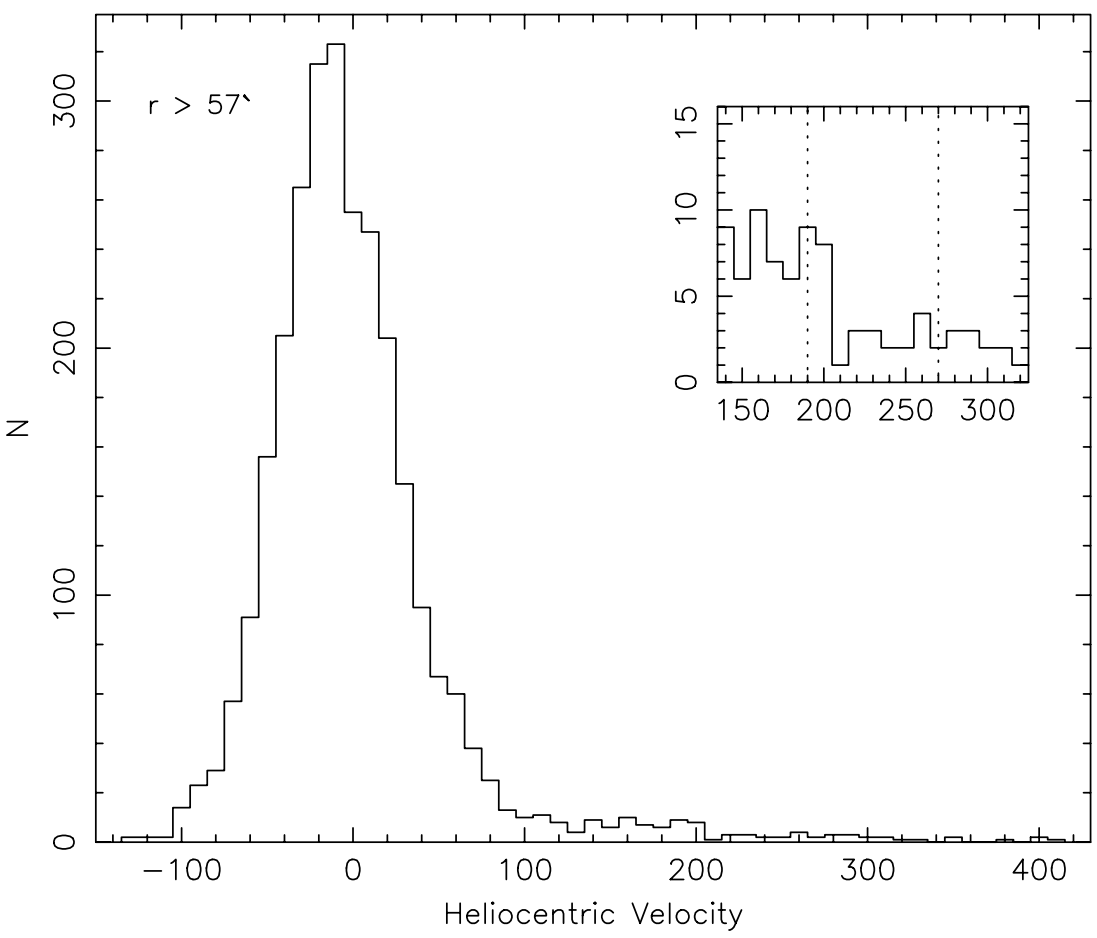

Figure 8. Velocity histogram for stars lying further than $57^{\prime}$, the adopted tidal radius, from the center of $\omega$ Cen. The inset panel shows the distribution near the heliocentric velocity of the cluster and the dotted lines outline a $\pm 40 \mathrm{~km} \mathrm{~s}^{-1}$ window about that value (see Figure 4).

Table 5

Candidate Extra-Tidal Cluster Members

\begin{tabular}{lccccccc}
\hline \hline ID & $\begin{array}{c}R \\
(\operatorname{arcmin})\end{array}$ & $\begin{array}{c}\text { Velocity } \\
\left(\mathrm{km} \mathrm{s}^{-1}\right)\end{array}$ & $N$ & $\begin{array}{c}\text { R.A. } \\
(\mathrm{J} 2000)\end{array}$ & $\begin{array}{c}\text { Decl. } \\
(\mathrm{J} 2000)\end{array}$ & $V$ & $V-I$ \\
\hline 4_7_288 & 66.5 & 238 & 1 & 132203.5 & -464237 & 16.43 & 0.98 \\
7_8_1878 & 70.1 & 238 & 1 & 132007.1 & -470950 & 15.70 & 1.28 \\
14_3_2302 & 93.0 & 246 & 1 & 132749.7 & -490100 & 16.57 & 1.08 \\
1_2_745 & 99.0 & 230 & 1 & 132342.4 & -455446 & 15.90 & 1.18 \\
15_5_941 & 112.6 & 219 & 1 & 133036.3 & -491428 & 16.50 & 1.02 \\
3_8_141 & 113.3 & 227 & 1 & 133101.3 & -454408 & 16.42 & 1.01
\end{tabular}

approximately the magnitude of the red clump, which is not inconsistent with the proposed interpretation.

For the nine stars with line strengths consistent with cluster membership, two have velocities of 192 and $195 \mathrm{~km} \mathrm{~s}^{-1}$, respectively, while a third has $V_{r}=261 \mathrm{~km} \mathrm{~s}^{-1}$. The remaining six have a mean velocity of $233 \mathrm{~km} \mathrm{~s}^{-1}$, corresponding closely to that for the cluster, and a dispersion of only $9 \mathrm{~km} \mathrm{~s}^{-1}$, which given the velocity errors is consistent with an intrinsic dispersion of near zero. These are exactly the characteristics expected for an extra-tidal population and we tentatively identify these six stars as likely extra-tidal (former) cluster members. Table 5 lists their properties. The spectrum of one of the stars is shown in the bottom panel of Figure 2. Given the small number and the shape of the region sampled (see Figure 3), no inferences can be made from the spatial distribution of these stars, except to note that the furthest lies almost exactly twice the adopted tidal radius from the cluster center (which is also the approximate limit of the area surveyed) and that the surface density corresponds to 1.2 stars per square degree for the region between 1 and 2 tidal radii. Clearly more accurate velocities and abundance information are required to more firmly establish the association or otherwise of these stars with $\omega$ Cen, but for the present we will assume that they are all extra-tidal members of the cluster and treat derived quantities as upper limits. The alternative assumption that all or some of these stars are simply members of the field halo population cannot be ruled out.

The immediate question to ask is what fraction of the mass of $\omega$ Cen is potentially represented by the extra-tidal stars. Assuming that the extra-tidal population is identical with that of the cluster, we can estimate the fraction as follows. Using a core radius of $2.4^{\prime}$ and a tidal radius of $57^{\prime}$ and the integral formulae given in King (1962), the percentage of the total luminosity of the cluster contained between $20^{\prime}$ and the tidal radius is approximately $10 \%$. Assuming that mass follows light, this figure also represents the fraction of the total mass contained in this radial region. The region is also characterized by the 154 likely members we have identified, whereas using the surface density calculated above, the region between 1 and 2 tidal radii is estimated to contain, at most, approximately ten extratidal members with the same characteristics. Consequently, combining these numbers, we conclude that an upper limit on the amount of mass in extra-tidal material lying between 1 and 2 tidal radii from the cluster center is of order $0.7 \pm$ $0.2 \%$ of the total cluster mass. It may well be considerably less. The uncertainty quoted comes solely from assuming that Poisson statistics apply to the sample of six identified extratidal member candidates. This limit is comparable to the results of Kiss et al. (2007) who estimated an $\sim 1 \%$ upper limit on the extra-tidal populations of three globular clusters (NGC 288, M30, and M55) but differs from that of Grillmair \& Johnson (2006) who estimate that at least $3 \%$ of the present cluster mass is associated with the tidal tails of the globular cluster NGC 5466. The biggest contrast is of course with Pal 5, where the mass in the extensive tidal tails exceeds the mass of the cluster itself (e.g., Odenkirchen et al. 2003).

\subsection{The Surface Density Profile}

In Figure 9 we show the (circularly averaged) surface density profile of $\omega$ Cen from the center to the outermost regions, 


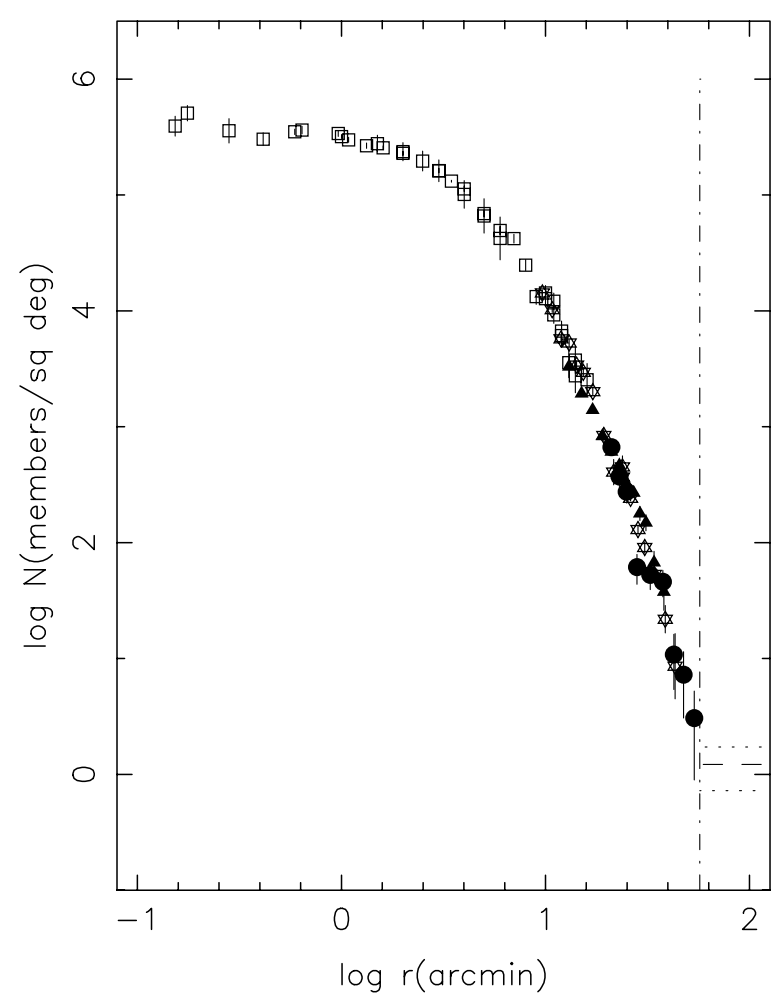

Figure 9. The (circularly averaged) surface density profile for $\omega$ Cen. Open squares and star symbols are surface photometry and star-count data, respectively, from Da Costa (1979). The filled triangles are data from M. G. Coleman \& G. S. Da Costa (2008, in preparation) while the filled circles are derived from the cluster member sample determined in this paper. The vertical scale corresponds to the surface density of stars in the cluster member sample. It can be converted to the surface brightness scale of Da Costa (1979) by subtracting 8.318. The vertical dot-dash line indicates the adopted tidal radius. The horizontal dashed line shows the surface density inferred for the region between 1 and 2 tidal radii if the six candidate extra-tidal stars are indeed associated with the cluster. The dotted lines represent the statistical uncertainty in that density.

covering a factor of $\sim 10^{5}$ in surface density. The profile combines the surface photometry and star-count data from Da Costa (1979), ${ }^{5}$ together with the circularly averaged surface density data from M. G. Coleman \& G. S. Da Costa (2008, in preparation) and that derived from our cluster member sample. The points from M. G. Coleman \& G. S. Da Costa (2008, in preparation) are for stars from just below the turnoff and brighter (see Figure 1) and are for regions where image crowding is not a concern. The background density was determined from the extensive region beyond the tidal radius covered in the imaging survey. The stars included correspond to those that dominate the integrated light, and thus no systematic effects are expected in scaling the surface density count data to the surface brightness observations (Da Costa 1979). In any case the long two-body relaxation time of $\omega$ Cen (e.g., Harris 1996), especially in the outer parts, means that significant mass segregation in this cluster is unlikely. Indeed van de Ven et al. (2006) find no dynamical evidence for any change in $M / L$ with radius, as expected given the relatively long relaxation time.

The surface density points from the cluster member sample were determined as follows. First, we grouped the radial distribution of the 154 probable $\omega$ Cen members into annuli. Mean

\footnotetext{
5 These data differ insignificantly from those of Meylan (1987) who adopted a very slightly different normalization of the star count data to the surface photometry.
}

Table 6

$\omega$ Cen Surface Density Data

\begin{tabular}{lccc}
\hline \hline $\begin{array}{l}\log r \\
(\operatorname{arcmin})\end{array}$ & $\left.\begin{array}{c}\log \mathrm{SD} \\
(10.0 \mathrm{~V} \mathrm{mag} \mathrm{arcmin}\end{array} \mathrm{mag}^{-2}\right)$ & $\begin{array}{c}\log r \\
(\operatorname{arcmin})\end{array}$ & $\begin{array}{c}\log \mathrm{SD} \\
\left(10.0 \mathrm{~V} \mathrm{mag} \operatorname{arcmin}^{-2}\right)\end{array}$ \\
\hline 1.115 & $-2.076 \pm 0.011$ & 1.323 & $-2.769 \pm 0.063$ \\
1.177 & $-2.308 \pm 0.014$ & 1.362 & $-3.022 \pm 0.080$ \\
1.231 & $-2.449 \pm 0.016$ & 1.398 & $-3.165 \pm 0.090$ \\
1.279 & $-2.674 \pm 0.022$ & 1.448 & $-3.806 \pm 0.129$ \\
1.323 & $-2.809 \pm 0.026$ & 1.513 & $-3.871 \pm 0.115$ \\
1.362 & $-2.928 \pm 0.031$ & 1.575 & $-3.933 \pm 0.115$ \\
1.398 & $-3.090 \pm 0.040$ & 1.629 & $-4.561 \pm 0.239$ \\
1.432 & $-3.164 \pm 0.044$ & 1.677 & $-4.734 \pm 0.286$ \\
1.463 & $-3.343 \pm 0.061$ & 1.729 & $-5.108 \pm 0.383$ \\
1.492 & $-3.422 \pm 0.069$ & $\cdots$ & $\cdots$ \\
1.532 & $-3.766 \pm 0.097$ & $\ldots$ & $\cdots$ \\
1.589 & $-4.019 \pm 0.160$ & $\cdots$ & $\cdots$ \\
\hline
\end{tabular}

Notes. The first two columns are from M. G. Coleman \& G. S. Da Costa (2008, in preparation); the remainder is from the cluster member sample determined here. The surface densities have the same normalization as Table 1 of Meylan (1987). Add 0.832 (equivalent to a central surface brightness of $16.81 \mathrm{~V}$ (mag $\operatorname{arcsec}^{-2}$ ) for $\log$ (surface density) values in the adopted units).

surface densities were then calculated with errors determined from the number of stars in each annulus. While Figure 3 shows that each annulus has not been completely surveyed, we assume that there is no radial variation in the degree of incompleteness. The resulting surface densities were then matched with the existing data in the region between $20^{\prime}$ and $26^{\prime}$. As is evident from Figure 9, the agreement between the new and existing data at these and larger radii is excellent. This serves as confirmation that our sample, while not complete, is suitably representative. Overall the profile shows a rapidly declining surface density distribution with a limiting radius consistent with the value we have adopted for the cluster tidal radius, shown by the vertical line in the figure. Figure 9 also shows the density inferred for the extra-tidal population in the region between 1 and 2 tidal radii, if the six candidates identified here are all associated with the cluster.

Given the extensive range in surface density, the complete profile shown in Figure 9, not surprisingly, cannot be adequately represented by analytic formulae such as those of King (1962), or by simple models (e.g., King 1966). However, more complex models, for example those of Meylan (1987), Meylan et al. (1995), and Merritt et al. (1997), do appear capable of fitting the entire profile. We note in passing that the models of van de Ven et al. (2006) adopt a tidal radius of $45^{\prime}$, which on the basis of Figure 9 is somewhat too small. The core radius of the cluster is well established at $r_{c} \approx 2.5^{\prime}$ (e.g., King 1962; Da Costa 1979; Trager et al. 1995). Table 6 lists the surface density data from M. G. Coleman \& G. S. Da Costa (2008, in preparation) and from this work in a manner that is directly compatible with Table 1 of Meylan (1987).

\section{DISCUSSION}

The photometric work of Law et al. (2003) (also see Coleman, M. G., \& Da Costa, G. S. 2008, in preparation) concluded that $\omega$ Cen lacks any notable extra-tidal structure, with the extensive tidal tails originally proposed by Leon et al. (2000) arising from the lack of allowance for the effects of reddening variations across the field (a possibility that Leon et al. 2000 themselves acknowledged). Our spectroscopy-based results are consistent with, and strengthen, this result. 
As noted in Section 1, the presence of extra-tidal structures, particularly in the form of tidal tails, is relatively well established in a number of globular clusters (e.g., Grillmair et al. 1995; Leon et al. 2000; Grillmair \& Johnson 2006, and the references therein) with the most striking example being Pal 5 (Odenkirchen et al. 2003; Grillmair \& Dionatos 2006). Clearly, however, $\omega$ Cen does not fall into this category. The most likely explanation for this is as follows. The largest dynamical influence on $\omega$ Cen is the "tidal shocking" that occurs each time the cluster passes through the Galactic plane (e.g., van de Ven et al. 2006), as is also the case for Pal 5 (cf. Dehnen et al. 2004). van de Ven et al. (2006) present a detailed model of the structure of $\omega$ Cen, constrained by high-quality proper motions and radial velocities for many thousands of cluster member stars, and we can use this model to estimate the effects of tidal shocks on the outer parts of the cluster. With the parameters given in section 9.5 of van de Ven et al. (2006), which rely on the $\omega$ Cen orbital parameters listed by Dinescu et al. (1999b), the average change in the velocity of a $\omega$ Cen star as a result of the impulse that occurs as the cluster traverses the Galactic plane is estimated as $|\Delta v| \approx 0.17 r^{\prime} \mathrm{km} \mathrm{s}^{-1}$, where $r^{\prime}$ is the radial distance of the star from the cluster center in arcmin. This is to be compared with the local escape velocity, which can be approximated by twice the local velocity dispersion (e.g., Binney \& Tremaine 1987, p. 490). The form of the velocity dispersion in the outer parts of $\omega$ Cen is not well established (even the observations of Scarpa et al. 2003 reach only a maximum of $\sim 24^{\prime}$ from the center), but it is clear that it is only in the outermost parts of the cluster that the tidal shock effects are large enough to add sufficient energy to (some) cluster stars to cause them to escape. Indeed as van de Ven et al. (2006) note, the phase-space structure of the outer parts of $\omega$ Cen may well be completely dominated by the effects of the relatively frequent tidal shocks: van de Ven et al. (2006) estimate that the cluster is immersed in the disk and feels the additional gravitational field for $\sim 10 \%$ of the $\sim 120$ Myr orbital period. The main point here though is that the region of $\omega$ Cen which is most strongly influenced by the tidal shocks contains only a very small fraction of the total mass of the cluster, unlike the situation for Pal 5 (Dehnen et al. 2004). For example, only $\sim 1 \%$ of the $\omega$ Cen mass lies between $\sim 40^{\prime}$ and the tidal radius. Consequently, it is likely that tidal shocks are indeed driving the escape of stars consistent with the limits on the extra-tidal population observed here. Further, we note that if stars leave the cluster with a relative velocity of $\sim 1 \mathrm{~km} \mathrm{~s}^{-1}$, then it is sufficient for the star to traverse the region between 1 and 2 tidal radii in less than the orbital period of the cluster around the Galaxy. Thus even if tidal shocks are driving the escape of a small number of stars, the number remaining close to the cluster will be small, again consistent with the observed lack of extra-tidal stars in our study. Indeed this suggests that if it were possible to isolate putative $\omega$ Cen main-sequence stars at and beyond the tidal radius against the enormous field contamination, it may well be possible to characterize tidal tails from $\omega$ Cen. Such discrimination might be possible with GAIA data though many square degrees of sky would need to be searched.

In the context of the model in which $\omega$ Cen is the remnant nucleus of a disrupted former dwarf galaxy, it is clear from the above that any stars which are more loosely bound than those in the outer parts of the remnant nucleus/star cluster will be even more strongly affected by the tidal shocks. Consequently, they would have been lost from the vicinity of the remnant nucleus/star cluster at relatively early stages in the interaction, once the disrupting dwarf galaxy has approached the current orbit of $\omega$ Cen. The dynamical models of Bekki \& Freeman (2003) and Tsuchiya et al. (2003) show that this occurs within about $3 \mathrm{Gyr}$ of the initiation of the interaction. Given that any gas in the original dwarf would have been used up in star formation (Bekki \& Freeman 2003) (and any remaining stripped out) in the first phases of the interaction, and that $\omega$ Cen contains only stars with ages in excess of $\sim 10 \mathrm{Gyr}$ (e.g., Sollima et al. 2005; Stanford et al. 2006), it is evident that the more loosely bound stars have been lost from the vicinity of $\omega$ Cen many Gyr ago, and are now widely dispersed around the Galaxy. The kinematic signatures of local field stars that may have had their origin with the disrupted system that once contained $\omega$ Cen have been modeled, and such stars may well have been observationally detected (e.g., Dinescu 2002; Mizutani et al. 2003; Chiba \& Mizutani 2004; Meza et al. 2005).

In this sense the currently disrupting dwarf galaxy Sagittarius (Sgr) and its central massive star cluster M54 provide an example similar to the postulated scenario for $\omega$ Cen, but on a longer timescale - eventually the Sgr field stars will disperse uniformly around the Galaxy but like $\omega$ Cen, M54 will retain its dynamical integrity for a very long time.

\section{CONCLUSIONS}

We have carried out an extensive radial velocity and linestrength analysis of observations of a large sample of stars that covers the outer parts of the globular cluster $\omega$ Cen and a substantial area beyond the cluster's tidal radius. While the analysis has led to the establishment of cluster membership for $\sim 150$ stars in the outer parts of the cluster, and revealed that the steeply declining radial surface density distribution of these stars is consistent with other extant data, the survey has identified only a very small number of objects that can be plausibly classified as extra-tidal cluster stars. The upper limit on the amount of mass potentially contained in the region between 1 and 2 tidal radii is less than $1 \%$ of the present cluster mass. We therefore conclude that there is no compelling evidence for any significant extra-tidal population associated with $\omega$ Cen.

The authors are grateful for the excellent support at the telescope provided by the staff of the Anglo-Australian Observatory. The comments of the anonymous referee led to improvements in the clarity of the paper. This research has made use of the SIMBAD database, operated at CDS, Strasbourg, France.

Facilities: AAT (2dF), ANU Siding Spring Observatory $1 \mathrm{~m}$ telescope

\section{REFERENCES}

Bedin, L. R., Piotto, G., Anderson, J., Cassisi, S., King, I. R., Momany, Y., \& Carraro, G. 2004, ApJ, 605, L125

Beers, T. C., Rossi, S., Norris, J. E., Ryan, S. G., \& Shefler, T. 1999, AJ, 117, 981 Bekki, K., \& Freeman, K. C. 2003, MNRAS, 36, L11

Binney, J., \& Tremaine, S. 1987, Galactic Dynamics (Princeton, NJ: Princeton Univ. Press)

Chiba, M., \& Mizutani, A. 2004, PASA, 21, 237

Coleman, M. G., Da Costa, G. S., Bland-Hawthorn, J., \& Freeman, K. C. 2005a, AJ, 129, 1443

Coleman, M. G., Da Costa, G. S., \& Bland-Hawthorn, J. 2005b, AJ, 130, 1065 Da Costa, G. S. 1979, AJ, 84, 505

Dehnen, W., Odenkirchen, M., Grebel, E. K., \& Rix, H.-W. 2004, AJ, 127, 2753

Dinescu, D. I. 2002, in ASP Conf. Ser. 265, Omega Centauri, A Unique Window into Astrophysics, ed. F. van Leeuwen, J. D. Hughes, \& G. Piotto (San Francisco, CA: ASP), 365

Dinescu, D. I., van Altena, W. F., Girard, T. M., \& López, C. E. 1999a, AJ, 117,277 
Dinescu, D. I., Girard, T. M., \& van Altena, W. F. 1999b, AJ, 117, 1792

Freeman, K. C. 1993, in ASP Conf. Ser. 48, The Globular Cluster-Galaxy Connection, ed. G. H. Smith, \& J. P. Brodie (San Francisco, CA: ASP), 608

Freeman, K. C., \& Rodgers, A. W. 1975, ApJ, 201, L71

Grillmair, C. J., \& Dionatos, O. 2006, ApJ, 643, L17

Grillmair, C. J., \& Johnson, R. 2006, ApJ, 639, L17

Grillmair, C. J., Freeman, K. C., Irwin, M., \& Quinn, P. J. 1995, AJ, 109, 2553

Hambly, N. C., Davenhall, A. C., Irwin, M. J., \& MacGillivray, H. T. 2001, MNRAS, 326, 1315

Harris, W. E. 1996, AJ, 112, 1487

King, I. 1962, AJ, 67, 471

King, I. 1966, AJ, 71, 64

Kiss, L. L., Székely, P., Bedding, T. R., Bakos, G. Á., \& Lewis, G. F. 2007, ApJ, 659, L129

Law, D. R., Majewski, S. R., Skrutskie, M. F., Carpenter, J. M., \& Ayub, H. F. 2003, AJ, 126, 1871

Leon, S., Meylan, G., \& Combes, F. 2000, A\&A, 359, 907

Lewis, I. J., et al. 2002, MNRAS, 333, 279

Merritt, D., Meylan, G., \& Mayor, M. 1997, AJ, 114, 1074

Meylan, G. 1987, A\&A, 184, 144

Meylan, G., Mayor, M., Duquennoy, A., \& Dubath, P. 1995, A\&A, 303, 761

Meza, A., Navarro, J. F., Abadi, A., \& Steinmetz, M. 2005, MNRAS, 359, 93

Mizutani, A., Chiba, M., \& Sakamoto, T. 2003, ApJ, 589, L89

Norris, J. E. 2004, ApJ, 612, L25

Norris, J. E., \& Da Costa, G. S. 1995, ApJ, 447, 680
Norris, J. E., Freeman, K. C., Mayor, M., \& Seitzer, P. 1997, ApJ, 487, L187

Norris, J. E., Freeman, K. C., \& Mighell, K. J. 1996, ApJ, 462, 241

Odenkirchen, M., et al. 2003, AJ, 126, 2385

Pancino, E., Galfo, A., Ferraro, F. R., \& Bellazzini, M. 2007, ApJ, 661, L155

Pancino, E., Pasquini, L., Hill, V., Ferraro, F. R., \& Bellazzini, M. 2002, ApJ, 568, L101

Piotto, G., et al. 2005, ApJ, 621, 777

Romano, D., Matteucci, F., Tosi, M., Pancino, E., Bellazzini, M., Ferraro, F. R., Limongi, M., \& Sollima, A. 2007, MNRAS, 376, 405

Scarpa, R., Marconi, G., \& Gilmozzi, R. 2003, A\&A, 405, L15

Smith, M. G., \& Wing, R. F. 1973, PASP, 85, 659

Sollima, A., Pancino, E., Ferraro, F. R., Bellazzini, M., Straniero, O., \& Pasquini, L. 2005, ApJ, 634, 332

Stanford, L. M., Da Costa, G. S., \& Norris, J. E. 2007, ApJ, 667, 911

Stanford, L. M., Da Costa, G. S., Norris, J. E., \& Cannon, R. D. 2006, ApJ, 647, 1075

Trager, S. C., King, I. R., \& Djorgovski, S. 1995, AJ, 109, 218

Tsuchiya, T., Dinescu, D. I., \& Korchagin, V. I. 2003, ApJ, 589, L29

van de Ven, G., van den Bosch, R. C. E., Verolme, E. K., \& de Zeeuw, P. T. 2006, A\&A, 445, 513

van Loon, J. Th., van Leeuwen, F., Smalley, B., Smith, A. W., Lyons, N. A., McDonald, I., \& Boyer, M. L. 2007, MNRAS, 382, 1353

Zacharias, N., Urban, S. E., Zacharias, M. I., Wycoff, G. L., Hall, D. M., Monet, D. G., \& Rafferty, T. J. 2004, AJ, 127, 3043 